\title{
Review: Inertial Sensors for Low-Frequency Seismic Vibration Measurement
}

\author{
by C. Collette, S. Janssens, P. Fernandez-Carmona, K. Artoos, \\ M. Guinchard, C. Hauviller, and A. Preumont
}

\begin{abstract}
The objective of this paper is to review recent advances in the sensors used to measure seismic linear vibrations at low frequencies. The main types of inertial sensors are reviewed: absolute displacement sensors, geophones, accelerometers, and seismometers. The working principle of each of them is explained, along with the general strategies to extend their bandwidth. Finally, the principle fundamental limitations of all inertial sensors are reviewed: tilt-to-horizontal coupling, zerolength springs, and sources of noise.
\end{abstract}

\section{Introduction}

The measurement of low-frequency and smallamplitude seismic vibrations with a high accuracy is critical in two scientific disciplines: seismology and structural vibration control. In both fields, there is a constant necessity to develop sensors with a better resolution, robust to temperature variations and magnetic fields, with a low power consumption, and, of course, at an affordable price. This is particularly valid in frontier science facilities, such as future particle colliders and gravitational wave detectors.

The objective of this paper is to review the main sensor types used to measure small vibrations at low frequencies; our focus is restricted to linear vibrations. Readers interested in rotation sensors can find excellent reviews on this topic in Lee (2009). We first provide a few basic definitions, followed by a detailed treatment of specific sensors-geophones, accelerometers, broadband seismometers and displacement sensors. For each of these sensor types, the basic working principle is explained, along with the main performances and limitations. The paper finishes with a brief discussion on the modelling and measurement of the sensor noise.

\section{Definitions}

The sensitivity of a sensor, $S$, is the conversion factor relating the physical quantity to measure, $U$, and the output voltage signal, $V_{\mathrm{o}}$ :

$$
S=V_{\mathrm{o}} / U
$$

It depends on the frequency but is constant in the useful bandwidth of the sensor.

The instrumental noise of a sensor, $N$, is the part of the output signal that does not correspond to the physical quantity $U$. The origin can be either external (pressure, humidity, temperature, electrical networks, magnetic fields, radiations, etc.) or internal (Brownian motion of the mass, amplifier noise, and so on). To some extent, the effect of external sources can be reduced by shielding the sensor, but the level of internal noise is inherent to the choice of the sensor components. The main sources of internal noise are reviewed in the section Sources of Internal Noise. In general, the overall level of noise depends on the frequency and must be specified in $V / \sqrt{\mathrm{Hz}}$. Alternatively, it can also be specified by its root-mean-square (rms) value, $N_{\text {rms }}$, in a given frequency band, and expressed in $V$.

The resolution of a sensor, $R$, is the smallest physical quantity that the sensor can measure. It is defined as the ratio between the noise and the sensitivity. If the sensor measures displacement, the resolution is

$$
R(m / \sqrt{\mathrm{Hz}})=\frac{N(V / \sqrt{\mathrm{Hz}})}{S(V / m)} \quad \text { or } \quad R_{\mathrm{rms}}(m)=\frac{N_{\mathrm{rms}}(V)}{S(V / m)} .
$$

The dynamic range of a sensor (DR), usually expressed in decibels $(\mathrm{dB})$, is defined as the ratio between the maximum recordable output voltage (without saturation and nonlinearities) $V_{\mathrm{o}}^{\mathrm{max}}$ and the minimum output voltage (i.e., the instrumental noise $N_{\text {rms }}$ ) such that

$$
\mathrm{DR}=20 \log _{10}\left(V_{\mathrm{o}}^{\mathrm{max}} / N_{\mathrm{rms}}\right)
$$

or equivalently, by dividing numerator and denominator by $S$, as

$$
\mathrm{DR}=20 \log _{10}\left(U^{\max } / R_{\mathrm{rms}}\right),
$$

where $U^{\max }$ is the largest value of the quantity $U$ that the sensor can measure. To design a good sensor, a general objective is to maximize DR (i.e., to maximize $U^{\max }$ and minimize $U^{\max }$ ) in order to be able to measure both large and small values of $U$. 
Table 1 provides a list of definitions of the variables used in this paper.

\section{Absolute Measurement}

In order to understand absolute measurement, it is necessary to understand relative measurement with respect to an inertial reference. The sensor capable of such measurement, called an inertial sensor, provides the measurement of an absolute quantity (displacement, velocity, or acceleration) only in some frequency range. The basic principle of an inertial sensor is depicted in Figure 1. It consists of a seismic mass $m$, attached to the measurement point through a suspension, represented by a spring $k$ and a dashpot $c$.

The dynamic equation of the seismic mass is

$$
m \ddot{x}+c(\dot{x}-\dot{w})+k(x-w)=-m g,
$$

where $x$ is the displacement of the seismic mass, $w$ is the displacement of the support, and $g$ is the gravity constant. If we choose the origin of $x$ to have zero initial conditions (i.e., $x=0$ at the equilibrium point where the static deflection compensates the gravity), equation (5) becomes

$$
m \ddot{x}+c(\dot{x}-\dot{w})+k(x-w)=0 .
$$

Then, the measurement of $y=x-w$ is used to evaluate $w$. Writing equation (6) in function of $y$ gives

$$
m \ddot{y}+c \dot{y}+k y=-m \ddot{w} .
$$

Using the Laplace transform, equation (7) becomes

$$
m s^{2} Y+c s Y+k Y=-m s^{2} W,
$$

From equation (8), the transmissibility $T_{w y}(s)$ between the displacement of the attachment point $W(s)$ and the relative displacement $Y(s)$ is given by

$$
T_{w y}(s)=\frac{Y(s)}{W(s)}=\frac{-m s^{2}}{m s^{2}+c s+k} .
$$

The magnitude and phase of the frequency response $T_{w y}(j \omega)$ are shown in Figure 2 (dotted line). Above the resonance frequency of the oscillator $\omega_{0}=\sqrt{k / m}$, the measurement of the relative displacement $Y(s)$ is a perfect estimator of the displacement $W(s)$, because of the flat transfer function. This is the principle of an absolute displacement sensor. Similarly, if instead of the relative displacement $Y(s)$ we measure the relative speed $\dot{Y}(s)=s Y(s)$, it is a perfect estimator of the speed $\dot{W}(s)$ above $\omega_{0}$ (also dotted line in Fig. 2). This is the working principle of a geophone. The figure also shows the transmissibility $T_{\dot{w} y}$ between the speed of the support $\dot{W}(s)=s W(s)$ and $Y(s)$ (dashed line in Fig. 2) and the transmissibility $T_{\ddot{w} y}$ between the acceleration $\ddot{W}(s)=s^{2} W(s)$ and

\begin{tabular}{|c|c|}
\hline Variable & Definition \\
\hline$S$ & Sensitivity of a sensor \\
\hline$V_{\mathrm{o}}$ & Output voltage \\
\hline$U$ & Physical quantity to measure \\
\hline$N$ & Instrumental noise \\
\hline \multirow[t]{2}{*}{$R$} & - Resolution of a sensor in equation (2) \\
\hline & $\begin{array}{l}\text { - Resistance constant in equations (16), (18), (19), (20), } \\
\quad(24),(26) \text { and (47) }\end{array}$ \\
\hline$N_{\text {rms }}$ & Root-mean-square value of the sensor noise \\
\hline$N_{\text {rms }}$ & Root-mean-square value of the sensor noise \\
\hline$V_{\mathrm{o}}^{\max }$ & Maximum recordable voltage \\
\hline$D R$ & Dynamic range \\
\hline$m$ & Mass of the sensor inertial mass \\
\hline$k$ & Spring constant \\
\hline$c$ & Dashpot constant \\
\hline$w$ & Absolute displacement of the ground \\
\hline$g$ & $\begin{array}{l}\text { - Gravity constant in equations (5), (43), and (44) } \\
\text { - Gain of the controller in equations (12) and (14) }\end{array}$ \\
\hline$y$ & $\begin{array}{l}\text { Relative displacement between the inertial mass and the } \\
\text { support }\end{array}$ \\
\hline$Y$ & Laplace transform of $y$ \\
\hline$s$ & Laplace variable \\
\hline$T_{w y}$ & Transmissibility between $w$ and $y$ \\
\hline$\omega_{0}$ & Resonance frequency of the oscillator \\
\hline$\xi_{0}$ & Fraction of critical damping \\
\hline$k_{a}$ & Stiffness of the piezoelectric actuator \\
\hline$\delta$ & Elongation of the actuator \\
\hline$x_{a}$ & Displacement of the upper end point of the actuator \\
\hline$G$ & $\begin{array}{l}\text { - Sensitivity of the displacement sensor in } \\
\text { equations (13), (14), (33)-(39) } \\
\text { - Sensitivity converting voltage into acceleration in } \\
\text { equation (51) }\end{array}$ \\
\hline$i$ & Current in the feedback circuit \\
\hline$I$ & Laplace transform of $i$ \\
\hline$j$ & Imaginary number \\
\hline$T$ & $\begin{array}{l}\text { - Constant of the coil in equations (15)-(21), (35)-(39), } \\
(41) \\
\text { - Temperature in equations (45)-(47) }\end{array}$ \\
\hline$B$ & Magnetic field in the coil \\
\hline$L$ & Inductance of the coil \\
\hline$E(s)$ & Expression of the stretcher \\
\hline$\omega_{c}$ & Apparent corner frequency with the stretcher \\
\hline$\xi_{c}$ & Apparent fraction of critical damping with the stretcher \\
\hline$T_{s}$ & Constant of the part of the coil used as a sensor \\
\hline$T_{a}$ & Constant of the part of the coil used as an actuator \\
\hline$g_{p}, g_{i}, g_{d}$ & $\begin{array}{l}\text { Gains of the proportional-plus-integral-plus-derivative } \\
\text { (PID) controller }\end{array}$ \\
\hline$H$ & $\begin{array}{l}\text { - Expression of the PID controller in equations (25), } \\
\text { (34)-(36), (40), and (41) } \\
\text { - Ratio between } S_{2} \text { and } S_{1} \text { in equations (54)-(59) }\end{array}$ \\
\hline$Q$ & $\begin{array}{l}\text { Electric charges at the electrodes of the piezoelectric } \\
\text { transducer }\end{array}$ \\
\hline$C$ & Electrical capacitance of the piezoelectric transducer \\
\hline$V$ & $\begin{array}{l}\text { Voltage between the electrodes of the piezoelectric } \\
\text { transducer }\end{array}$ \\
\hline$d_{33}$ & Piezoelectric constant \\
\hline$n$ & Number of layers of piezoelectric material \\
\hline$f$ & $\begin{array}{l}\text { - Force exerted on the piezoelectric transducer in } \\
\text { equations (29) and (30) } \\
\text { - Frequency in equations (45)-(47) and (49)-(51) }\end{array}$ \\
\hline$C_{A}$ & Capacitance of the amplifier \\
\hline
\end{tabular}
$Y(s)$. Following the same reasoning, below $\omega_{0}, Y(s)$ is a perfect estimator of the acceleration $\ddot{W}(s)$ but is scaled by $1 / \omega_{0}^{2}$
Table 1

Nomenclature 


\section{Table 1 (Continued)}

\begin{tabular}{|c|c|}
\hline Variable & Definition \\
\hline$\theta$ & Tilt angle of the inertial sensor \\
\hline$\Theta$ & Laplace transform of $\theta$ \\
\hline$\Phi_{F}$ & $\begin{array}{l}\text { Power spectral density of the thermomechanical force } \\
\text { exerted on a mass }\end{array}$ \\
\hline$k_{B}$ & Boltzmann's constant \\
\hline$\Phi_{B}$ & $\begin{array}{l}\text { Power spectral density of the acceleration of a mass } \\
\text { subjected to a thermomechanical force }\end{array}$ \\
\hline$\Phi_{J}$ & Power spectral density of the thermoelectrical noise \\
\hline$R_{C}$ & Electrical resistance of a capacitor \\
\hline$\Phi_{s h}$ & Shottky noise \\
\hline$I_{d c}$ & $\begin{array}{l}\text { Average current due to the random motion of the electric } \\
\text { charges }\end{array}$ \\
\hline$\Phi_{V}$ & Power spectral density of the flicker noise \\
\hline$K$ & Constant parameter in the expression of the flicker noise \\
\hline$\alpha$ & Constant parameter in the expression of the flicker noise \\
\hline$\Phi_{n n}$ & Power spectral density of the instrument noise \\
\hline$\Phi_{s s}$ & Power spectral density of the signal \\
\hline$\Phi_{x x}$ & Power spectral density of $x$ \\
\hline$\Phi_{y y}$ & Power spectral density of $y$ \\
\hline$\Phi_{x y}$ & Cross power spectral density between $x$ and $y$ \\
\hline$\gamma_{x y}^{2}$ & Coherence between $x$ and $y$ \\
\hline$Z$ & Load at which the current noise is developed \\
\hline$S_{1}$ and $S_{2}$ & Sensitivities of two sensors \\
\hline$N_{1}$ and $N_{2}$ & Noise of two sensors \\
\hline$\beta$ & Signal-to-noise ratio \\
\hline
\end{tabular}

(solid line in Fig. 2). This is the working principle of an accelerometer.

\section{Absolute Displacement Sensor}

In active vibration isolation, the quantity of interest is the absolute displacement of the sensitive equipment. As is apparent from the Absolute Measurement section, above

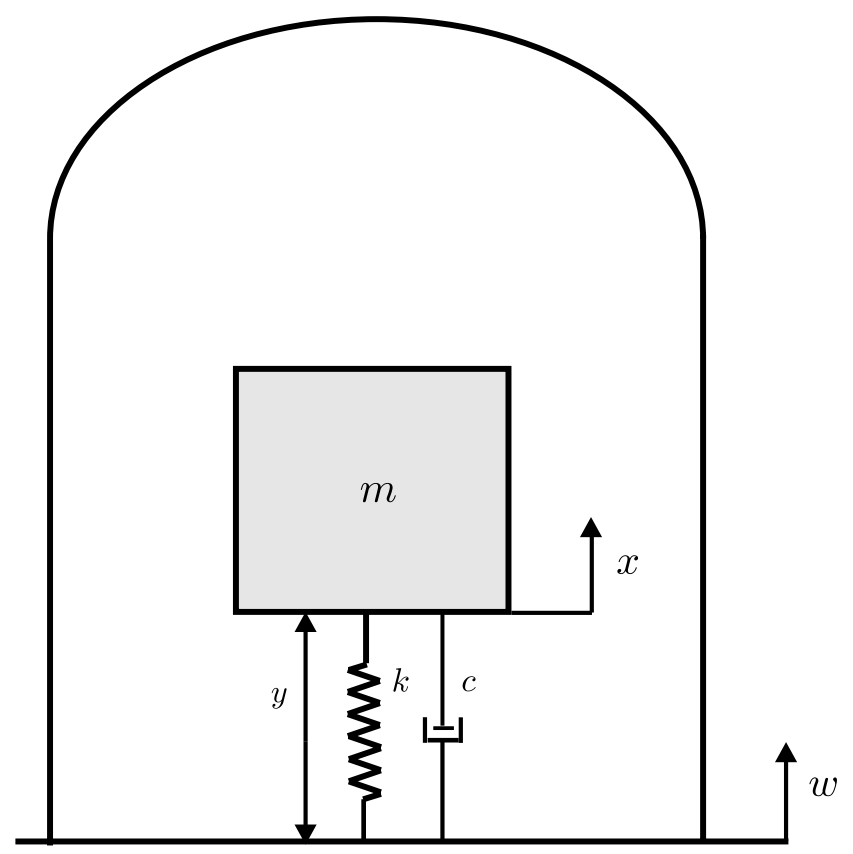

Figure 1. Working principle of an inertial sensor. (See Table 1 for definitions for all figures.)

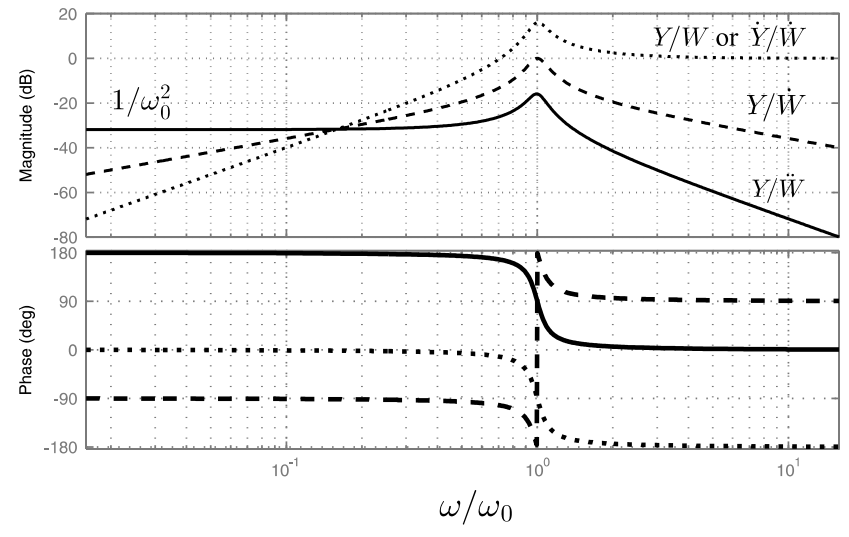

Figure 2. Transmissibilities of the sensor described in Figure 1.

the resonance of the seismic mass, the measurement of the relative displacement between the mass and the support provides this information. This section presents an active mean to extend the sensitivity of a displacement sensor in the lowfrequency range. The device is shown in Figure 3. To the best of our knowledge, there is no commercial product corresponding to this system.

It consists of a mass $m$ mounted on a soft element represented by a spring $k$ and a dashpot $c$ and has a relative displacement sensor $y$. In this case, the soft element is not directly fixed to the support, but to a piezoelectric actuator. In Figure $3, k_{a}(\gg k)$ is the stiffness of the actuator and $\delta$ its elongation.

The equation governing the dynamics of the seismic mass is

$$
m \ddot{x}+c\left(\dot{x}-\dot{x}_{a}\right)+k\left(x-x_{a}\right)=0,
$$

where $x$ and $x_{a}$ are as shown in Figure 3. The dynamic equilibrium of the point $x_{a}$ is expressed as

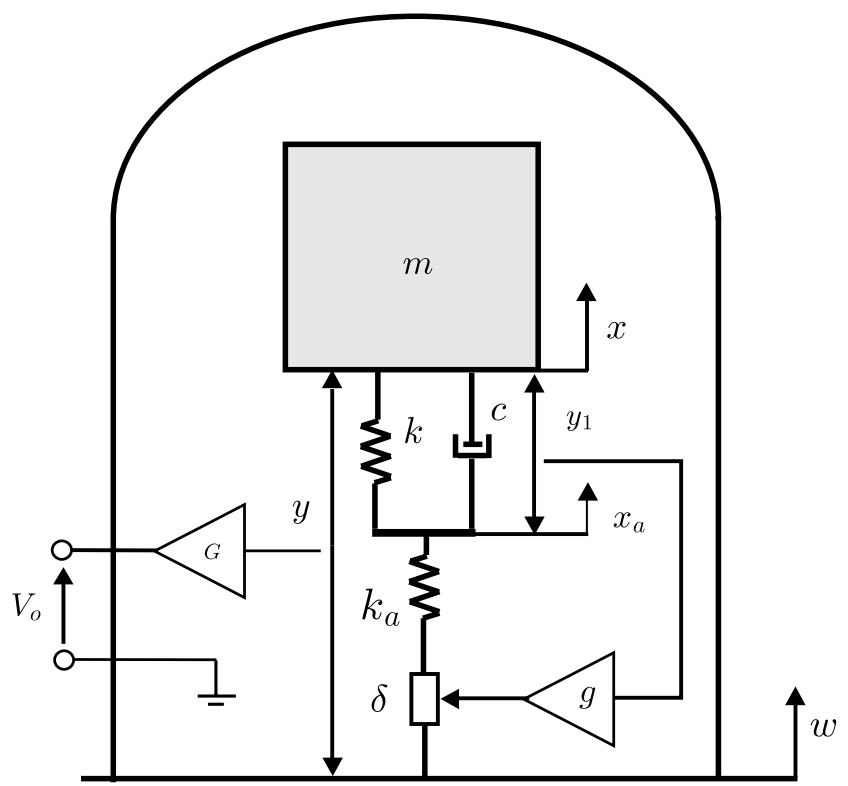

Figure 3. Working principle of an active displacement sensor (Fraanje et al., 2009). 
$c\left(\dot{x}-\dot{x}_{a}\right)+k\left(x-x_{a}\right)=k_{a}\left(x_{a}-w-\delta\right)$.

A second relative displacement sensor measures $y_{1}=x-x_{a}$, and the signal is fed back to the actuator in such a way that

$$
\delta=g y_{1}=g\left(x-x_{a}\right),
$$

where $g$ includes the gain of the controller and the sensitivity of the relative displacement sensor.

The output voltage of the sensor $V_{\mathrm{o}}$ is given by

$$
V_{\mathrm{o}}=G y=G(x-w) \text {. }
$$

By substituting equations (11)-(13) into equation (10), we get

$$
\frac{V_{\mathrm{o}}}{w}=-G \frac{\left(c m / k_{a}\right) s^{3}+m s^{2}\left(1+g+k / k_{a}\right)}{\left(c m / k_{a}\right) s^{3}+m s^{2}\left(1+g+k / k_{a}\right)+c s+k} .
$$

The typical sensitivity curve is shown in Figure 4. The feedback operation decreases the corner frequency from $\sqrt{k k_{a} /\left[m\left(k+k_{a}\right)\right]}$ to $\sqrt{k k_{a} /\left[m\left(k+(1+g) k_{a}\right)\right]}$.

\section{Geophone}

The working principle of the geophone is illustrated in Figure 5 (Roset et al., 2004). A coil is encircled around a seismic mass $m$ and loaded by a resistance $R$. The ground $w$ generates a relative motion between $m$ and the coil. The relative motion creates a current $i$ and a voltage $V_{\mathrm{o}}$ across the resistance.

The equations of the system are as follows:

$$
m \ddot{x}+c(\dot{x}-\dot{w})+k(x-w)+T i=0
$$

for the mechanical part and

$$
L \frac{d i}{d t}-T(\dot{x}-\dot{w})+R i=0
$$

for the electrical part, where $i$ is the current, $L$ is the inductance of the coil, and $T$ is the constant of the coil, expressed in $T m$ or $V /(m / s)$.

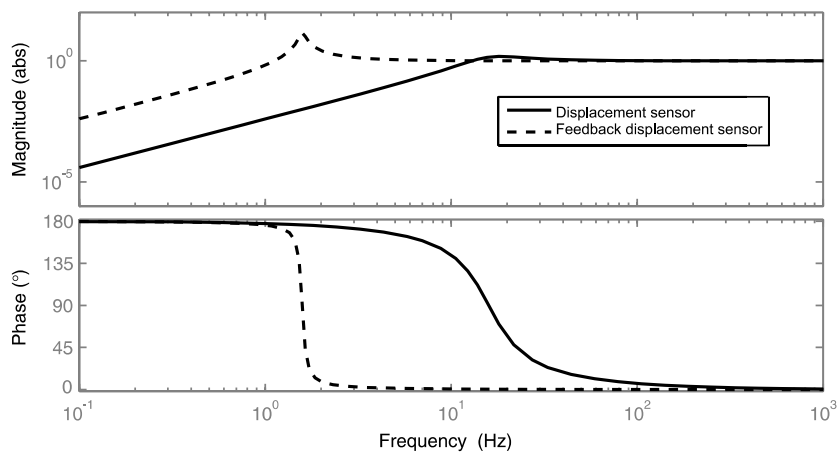

Figure 4. A typical sensitivity curve of a displacement sensor in an open-loop (solid line) and a closed-loop (dashed line) configuration. (a)

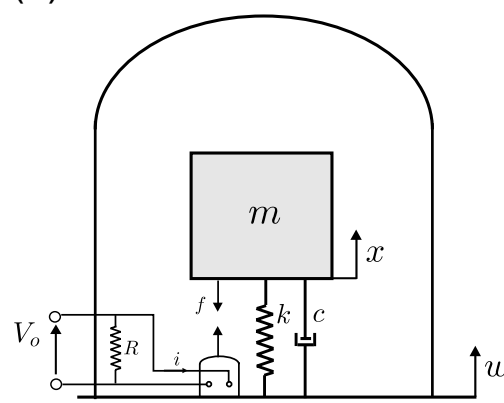

(b)

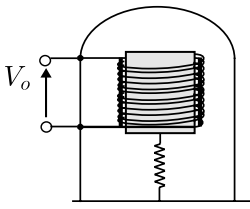

(c)

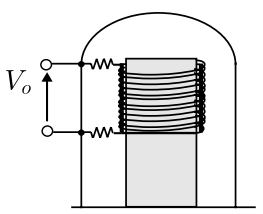

Figure 5. Electromagnetic geophone: (a) The model, and (b, c) two conceptual designs: a moving magnet topology (b) and a moving coil topology (c).

Defining $y=x-w$, in the Laplace domain we get

$$
m s^{2} Y+c s Y+k Y+T I=-m s^{2} W
$$

and

$$
L s I-T s Y+R I=0 .
$$

The output of the sensor is the voltage $V_{\mathrm{o}}$ across the resistance $R$; that is, $V_{\mathrm{o}}=R I$. The sensitivity of the geophone is given by

$$
\frac{V_{\mathrm{o}}}{s W}=\frac{R T}{L s+R} \frac{-m s^{2}}{m s^{2}+s c+k+T^{2} s /(L s+R)} .
$$

In practice, $R \gg s L$, and equation (19) is reduced to

$$
\frac{V_{\mathrm{o}}}{s W}=\frac{-m T s^{2}}{m s^{2}+s\left(c+T^{2} / R\right)+k}
$$

or, equivalently, to

$$
\frac{V_{\mathrm{o}}}{s W}=\frac{-T s^{2}}{s^{2}+2 \xi_{0} \omega_{0} s+\omega_{0}^{2}},
$$

which is the typical expression of a high-pass filter (Fig. 6), where $\omega_{0}=\sqrt{k / m}$ and $\xi_{0}=\left(c+T^{2} / R\right) /\left(2 m \omega_{0}\right)$. Actually, a geophone can measure the velocity of the support, typically from a few hertz to a few hundred hertz. At high frequency, the performances are limited by the higherorder modes, discussed in Zero-Length Spring. At low frequency, the performances are limited by the fundamental resonance of the inertial mass.

There are at least three possibilities to extend the bandwidth of a geophone at low frequency. The first one is to add a capacitor in series with the resistance (Pazos et al., 2005). In this case, the corner frequency can be decreased by a factor of $1 / 4$ without any loss of sensitivity. The second possibility is to stretch the sensitivity curve so that it has an apparent corner frequency at $\omega_{c}$ lower than its mechanical resonance frequency $\omega_{0}$ (Zuo, 2004; Zuo and Nayfeh, 2004). 


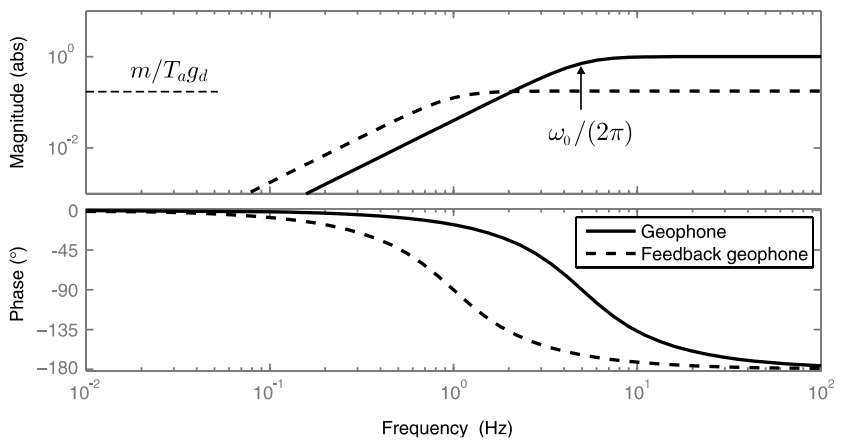

Figure 6. Typical sensitivity curve of a geophone in open-loop (solid line) and closed-loop (dashed line) configuration.

This can be directly implemented numerically by multiplying the output signal by

$$
E(s)=g \frac{s^{2}+2 \xi_{0} \omega_{0} s+\omega_{0}^{2}}{s^{2}+2 \xi_{c} \omega_{c} s+\omega_{c}^{2}},
$$

where $g$ is a constant gain and $\xi_{c}$ is the apparent damping. Because it is just a variable gain, the major limitation is that it does not improve the signal-to-noise ratio. Also, as the sensitivity curve is falling rapidly below the corner frequency, a digital implementation can result in a high additional quantization noise. To avoid this problem, $E(s)$ can be realized by an electrical circuit (Zuo and Nayfeh, 2004). A third possibility is to create a feedback geophone, as shown in Figure 7 (Preumont, 2006). becomes

Here, the coil is divided into two parts, and equation (17)

$$
m s^{2} Y+c s Y+k Y+T_{a} I_{a}+T_{s} I_{s}=-m s^{2} W
$$

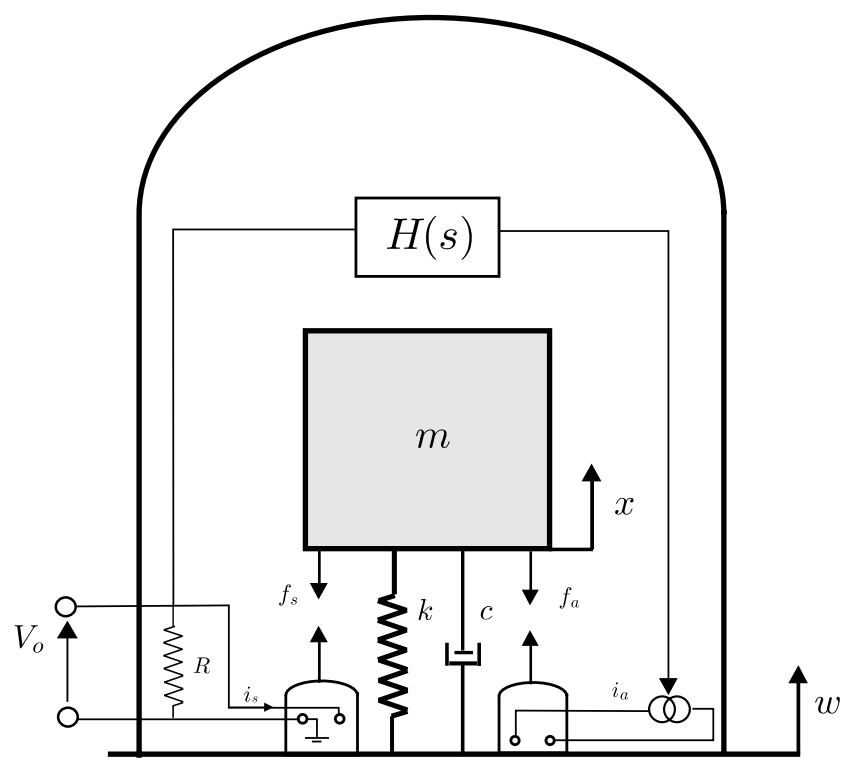

Figure 7. Working principle of a feedback geophone. where $T_{s}$ and $T_{a}$ are the constants of the two parts of the coil. One part is still used as a sensor. Using the same assumption that $R$ is large, the output voltage is given by

$$
V_{\mathrm{o}}=R I_{s} \simeq T_{s} s y
$$

where $I_{s}$ is the current generated by the relative motion between the mass and the ground. Then, $V_{\mathrm{o}}$ is used to generate a current in the other part of the coil. Taking a proportionalplus-integral-plus-derivative (PID) controller, we get

$$
I_{a}=H(s) V_{\mathrm{o}}=\left(g_{p}+g_{i} / s+s g_{d}\right) V_{\mathrm{o}},
$$

where $g_{p}, g_{i}$, and $g_{d}$ are the gains of the controller. Replacing equations (24) and (25) in equation (23) gives

$$
\frac{V_{\mathrm{o}}}{s W}=\frac{-m T_{s} s^{2}}{\left(m+T_{a} T_{s} g_{d}\right) s^{2}+\left(c+T_{s}^{2} / R+T_{a} T_{s} g_{p}\right) s+k+T_{a} T_{s} g_{i}} .
$$

The corner frequency of the geophone can be actively decreased from $\sqrt{k / m}$ to $\sqrt{\left(k+T_{a} T_{s} g_{i}\right) /\left(m+T_{a} T_{s} g_{d}\right)}$ by choosing a negative value for $g_{i}$ and a positive value for $g_{d}$. The proportional gain $g_{p}$ is chosen to adjust the damping. In the useful bandwidth, the sensitivity is reduced to

$$
\frac{V_{\mathrm{o}}}{s W}=\frac{-m T_{s}}{m+T_{a} T_{s} g_{d}},
$$

and the transfer function between the ground displacement and the relative displacement of the seismic mass is

$$
\frac{y}{W}=\frac{-m}{m+T_{a} T_{s} g_{d}} .
$$

As is apparent from equation (28), an additional feature of the relative acceleration feedback is that it forces the seismic mass to move with the ground, which reduces the relative displacement of the seismic mass. As a consequence, the sensor can measure much higher levels of vibrations without saturation, which is particularly useful to record strong earthquakes. This is known as the force-balance principle.

The typical sensitivity curve of a geophone in closedloop configuration is also shown in Figure 6 (dashed line). For even lower frequencies, force-balance accelerometers (FBA) or broadband seismometers are used (see ForceBalance Accelerometer and Seismometer section).

\section{Passive Accelerometer}

Broadband Accelerometer. A classical broadband accelerometer consists of a seismic mass fixed on a piezoelectric transducer, as shown in Figure 8. In this configuration, the transducer is of the stacked design, comprising $n$ layers of piezoelectric material. The equation governing the dynamics of the seismic mass is still given by equation (7), where $k$ and $c$ are now the equivalent stiffness and damping coefficients 


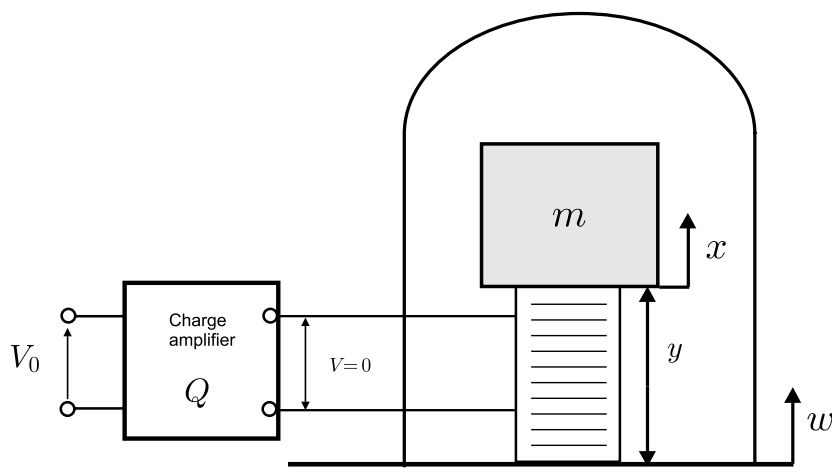

Figure 8. Working principle of a broadband accelerometer.

of the transducer with short-circuited electrodes. The constitutive equations of the piezoelectric transducer are

$$
\left(\begin{array}{l}
Q \\
y
\end{array}\right)=\left(\begin{array}{cc}
C & n d_{33} \\
n d_{33} & 1 / k
\end{array}\right)\left(\begin{array}{l}
V \\
f
\end{array}\right)
$$

with $d_{33}$ the piezoelectric constant, $Q$ the electric charge on the electrodes, and $V$ the voltage between them; $f$ the force exerted on the transducer and $y$ its extension; and $C$ its intrinsic electrical capacitance. The stack is connected to a charge amplifier, enforcing $V=0$. Using equation (29), the electric charge can be expressed as

$$
Q=n d_{33} f=n d_{33} k y,
$$

and the voltage at the output of the amplifier is given by

$$
V_{\mathrm{o}}=-Q / C_{A},
$$

where $C_{A}$ is the capacitance of the amplifier (Preumont, 2006). Combining equations (31), (30), and (7), we get

$$
\frac{V_{\mathrm{o}}}{s^{2} W}=\frac{n d_{33}}{C_{A}} \frac{m}{s^{2} / \omega_{0}^{2}+2 \xi / \omega_{0}+1},
$$

where $\omega_{0}$ is the resonance frequency of the seismic mass. These sensors are commonly used to measure structural vibrations, up to kilohertz. However, as their sensitivity is proportional to $m$, these sensors cannot resolve low-frequency seismic vibrations.

Seismic Accelerometer. The basic principle of a passive seismic accelerometer is described in Figure 1. The seismic mass is mounted on a compliant element, represented in the figure by $k$ and $c ; y$ is measured by a displacement transducer, giving a signal proportional to the acceleration, from DC up to the resonance frequency of the seismic mass (Liu and Kenny, 2001; Daku et al., 2004; Kollias and Avaritsiotis, 2005). For large values of $y$, nonlinearities appear in both the mechanical suspension and the displacement transducer, with the result that the dynamic range typically is limited to $100 \mathrm{~dB}$. This value can be extended by using a servo loop, as presented in the next section.
Force-Balance Accelerometer (FBA) and Seismometer

In a feedback accelerometer (Wienlandt, 2002), a coil is encircled around the seismic mass, as for a geophone, and a displacement sensor with a sensitivity $G$ is also used to measure $y$ (Fig. 9).

The output voltage of the sensor, $V_{0}$, is given by

$$
V_{\mathrm{o}}=G y .
$$

This signal, passing through a compensator $H(s)$, is also used to generate a current

$$
i=H(s) V_{\mathrm{o}}=H(s) G y,
$$

creating a force $f$ applied to the seismic mass and given by

$$
f=-T i=-T H(s) G y .
$$

Replacing equations (33) and (35) in equation (17), we get the expression of the sensitivity

$$
\frac{V_{\mathrm{o}}}{s^{2} W}=\frac{-m}{\left(m s^{2}+c s+k\right) / G+T H(s)} .
$$

Depending on $H(s)$, various behaviors can be obtained. If $H(s)=g_{p}$, equation (36) becomes

$$
\frac{V_{\mathrm{o}}}{s^{2} W}=\frac{-m}{\left(m s^{2}+c s+k\right) / G+T g_{p}} .
$$

The output $V_{\mathrm{o}}$ is proportional to the acceleration of the base in a large frequency range, from DC to the resonance of the system, which is extended by the servo loop from $\omega_{0}=\sqrt{k / m}$ to $\sqrt{\left(k+G T g_{p}\right) / m}$. In the useful bandwidth, the sensitivity is reduced to

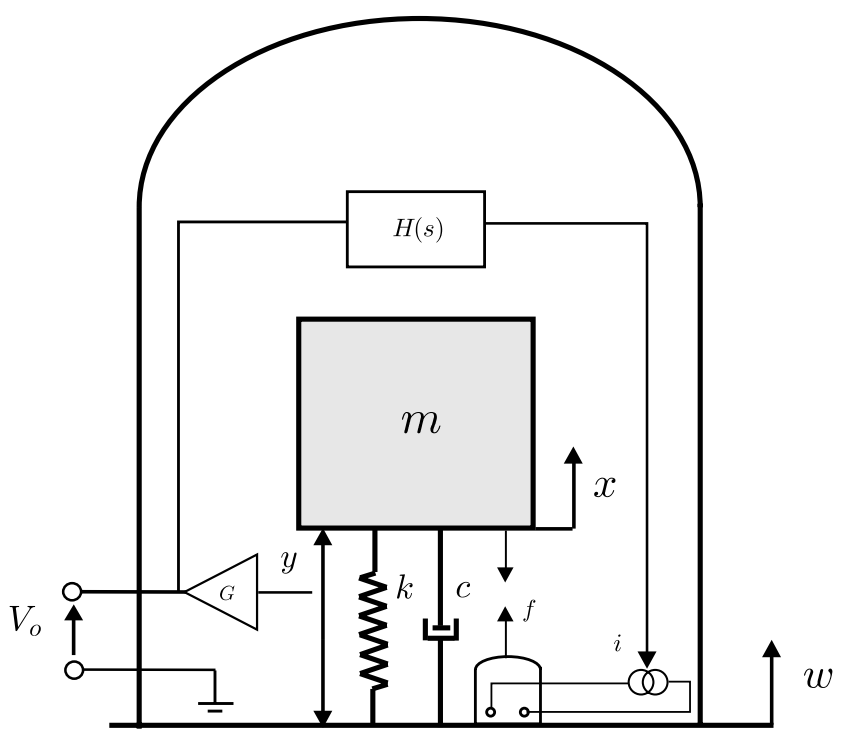

Figure 9. Working principle of a force-balanced sensor. 


$$
\frac{V_{\mathrm{o}}}{s^{2} w}=\frac{-m}{k / G+T g_{p}} \simeq \frac{-m}{T g_{p}},
$$

and the transfer function between the ground acceleration and the relative displacement of the seismic mass and the frame is

$$
\frac{y}{s^{2} W}=\frac{-m}{k+T G g_{p}},
$$

to be compared with equation (28). Here again, the feedback forces the seismic mass to move with the ground in the useful frequency range, renders the output linear, and increases the dynamic range. Now, the $V_{0}^{\max }$ in equation (3) is only limited by the electrical elements. This is also known as the forcebalance principle, and the sensor is an FBA.

In practice, an integrator is also added to remove the DC offset of the seismic mass, in which case

$$
H(s)=g_{p}+g_{i} / s,
$$

where $g_{i}$ is the gain of the integrator. The transmissibilities in open-loop and closed-loop configurations are, respectively, the solid and dashed lines shown in Figure 10.

An FBA can have a dynamic range of more than $150 \mathrm{~dB}$; that is, $50 \mathrm{~dB}$ more than a passive accelerometer.

FBAs are well adapted to record strong earthquakes, but it has been shown in Plesinger (1984) that, for a broadband record of the seismic activity, a sensor with a flat response to the ground velocity (i.e., a broadband seismometer) is better adapted (Sheffield, 1964; Melton and Johnson, 1962; Teupser and Plesinger, 1979; Usher et al., 1979; Wielandt and Streckeisen, 1982). This is achieved by introducing a derivative term in the controller, such as

$$
H(s)=g_{p}+g_{i} / s+s g_{d} .
$$

The value of $g_{d}$ is chosen to be the dominant term of the denominator in the useful frequency range of the sensor, leading to a flat response to the ground velocity, as shown in Figure 11. In the useful bandwidth, equation (36) is reduced to

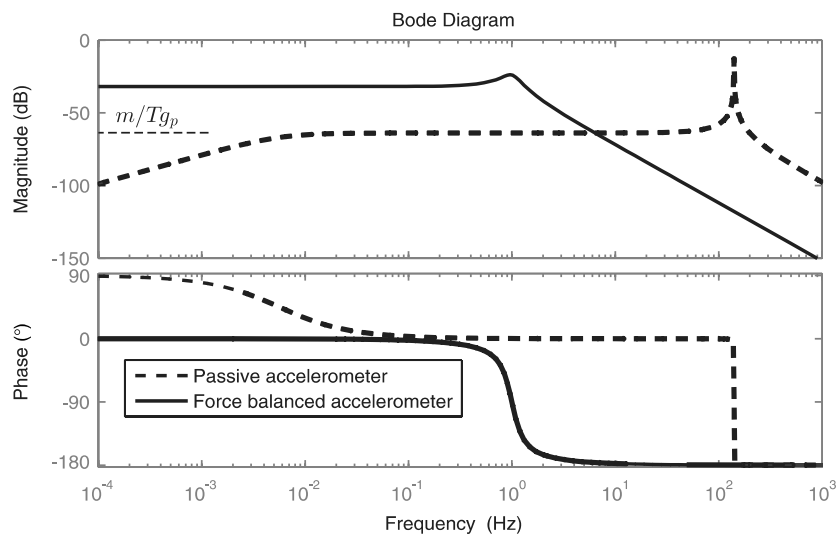

Figure 10. Typical sensitivity of an FBA (dashed line) compared to a passive seismic accelerometer (solid line).

$$
\frac{V_{\mathrm{o}}}{s w} \simeq \frac{m}{T g_{d}}
$$

Another class of seismometers is based on electrochemical transducers (e.g., EENTEC SP-400 and others). In this case, the oscillator is replaced by fluid (electrolytic solution) contained between elastic membranes. The transducer consists of a pair of electrodes plunged into the fluid. By a convective diffusion of the ions in the electrolyte, the motion of the fluid creates a current that is proportional to the fluid velocity. Compared to the devices described previously in this article, their main advantages are that they are immune to magnetic field, remain fully operable at high tilt, and have a low power consumption.

In addition to commercial products, several sensors have been developed for specific applications. For example, a high-sensitivity horizontal accelerometer has been developed for gravitational-wave interferometers (Bertolini et al., 2006a,b), and a modification of a geophone to produce a broadband seismometer has been described in Barzilai, VanZandt et al. (1998) and Barzilai (2000). Also, a nonmagnetic sensor has been developed in Frisch et al. $(2003,2004)$ to measure the mechanical vibrations of electromagnets in an accelerator environment. Finally, it should be noted that an optical technology can be used to measure $y$, such as reported in Gardner et al. (1987) and Zumberge et al. (2010).

\section{Tilt-to-Horizontal Coupling}

In the previous sections, we have considered only vertical inertial sensors. Figure 12 shows the more general configuration of an inclined sensor.

In this case, equation (5) becomes

$$
m \ddot{x}+c(\dot{x}-\dot{w})+k(x-w)=-m g \cos \theta,
$$

where $\theta$ is the tilt angle. Linearizing the equation around a nominal angle $\theta_{0}$ and again choosing zero initial conditions, in the Laplace domain we obtain

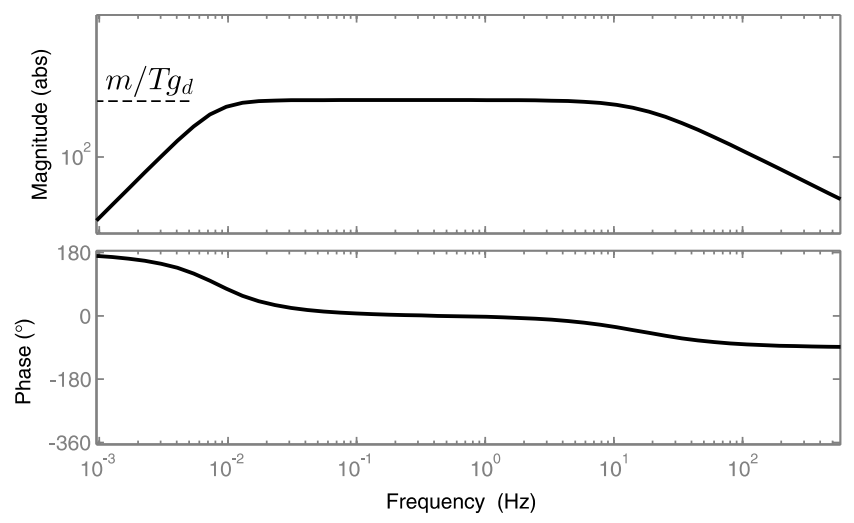

Figure 11. Typical sensitivity curve of a broadband seismometer. 


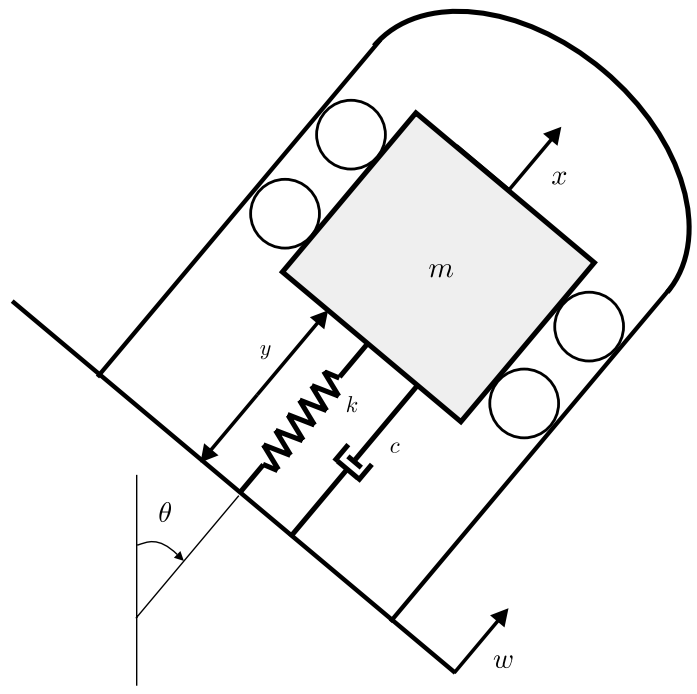

Figure 12. Tilted inertial sensor.

$$
Y(s)=\frac{-m s^{2}}{m s^{2}+c s+k} W(s)+\frac{-m g \sin \left(\theta_{0}\right)}{m s^{2}+c s+k} \Theta(s),
$$

which means that an inertial sensor cannot distinguish between a displacement of the support and a modification of the orientation with respect to the gravitational field. More precisely, $Y(s)$ is a measure the displacement of the support above the resonance of the mass and a measure of the tilt of the support below the resonance. The coupling is maximum for $\theta_{0}=\pi / 2$ and is known as tilt-to-horizontal coupling (Rodgers, 1968, 1969). This explains why stretching filters cannot be used for horizontal sensors. In order to minimize this problem, Rijnveld and Van Den Dool (2010) and Laro et al. (2011) present a solution in which the rotation between the sensor and the support is permitted. Thus, the sensor is not tilted when the support rotates and can still measure only the horizontal displacement.

\section{Zero-Length Spring}

In all of the inertial sensors described thus far in this article, the working principle relies on the availability of an oscillator with a very low resonance frequency, typically around $1 \mathrm{~Hz}$. In the first seismometers, it was realized by a mass suspended by a soft spring. The major drawback was that, as the static deflection increases with a decreasing resonance frequency, the size became rapidly prohibitive. In LaCoste (1934), a more compact design was proposed, represented in Figure 13a and Figure 13b for the vertical and the lateral directions, respectively.

Further decreases in size have been achieved with the introduction of leaf springs, which basically consist of prebent flexible beams (Wielandt and Streckeisen, 1982). This solution is still widely used in most of the geophones and seismometers. The major drawback of leaf springs is that
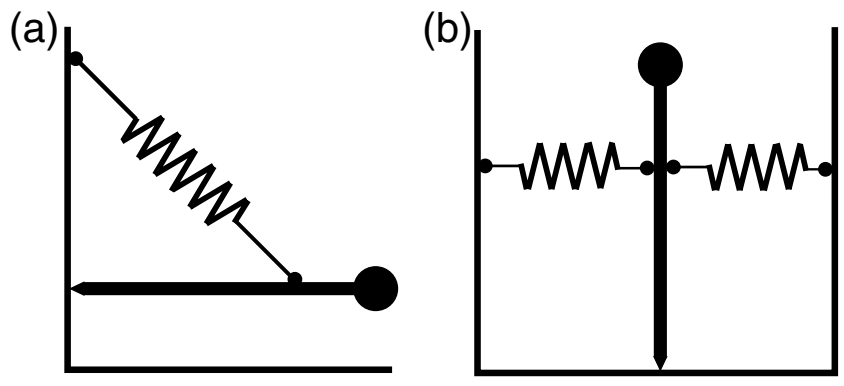

Figure 13. LaCoste suspensions (LaCoste, 1934).

the decrease in size is achieved at the cost of the introduction of spurious resonances (Ling et al., 1990; Faber and Maxwell, 1997), thereby requiring a careful design. Typically, an oscillator with a fundamental resonance at a few hertz has a second resonance between 100 and $200 \mathrm{~Hz}$. In Acernese et al. (2008, 2010), an interesting alternative has been presented, whereby a horizontal seismometer has been developed using a folded pendulum. It uses flexural joints and a tunable fundamental frequency, strongly decoupled from the higher-order modes. Other interesting devices are presented in Cacho et al. (1999), in which the rotative inertial mass has a $V$-shape to improve the sensitivity; Bertolini et al. (1999, 2004), in which a low-frequency oscillator is achieved with an antispring; and Oome et al. (2009), in which a permanent magnet is used to compensate for the gravitational force.

\section{Sensor Noise}

A low-noise floor is important to improve the resolution of a sensor (see equation 2). This is particularly critical when they are used in active vibration isolation strategies that do not rely on passive isolation (i.e. active hard mounts; Collette et al., 2010; van der Poel et al., 2007; van der Poel, 2010; Montag, 1996a). The main sources of noise are reviewed in the next section, followed by a general method to experimentally evaluate the sensor noise.

\section{Sources of Internal Noise}

Ground-motion sensors are all based on the measurement of the relative motion of a proof mass with respect to the ground. In such sensors, there are basically four different sources of noise (Rodgers, 1992, 1993,; Rodgers et al., 1995; Barzilai, VanZandt, and Kenny, 1998; Pallas-Areny and Webster, 2001): (1) thermomechanical noise, (2) thermoelectric noise, (3) shot noise, and (4) flicker noise.

Thermomechanical noise, or Brownian noise, of the seismic mass is caused by molecular collisions with air (Saulson, 1990; Rowan et al., 2005). This surrounding environment exerts a random force on the mass, $\Phi_{F}(\omega)$, for which the power spectral density is given by Nyquist's formula (Kittel, 1958; Saulson, 1990), 


$$
\Phi_{F}(f)=4 k_{B} T c\left(N^{2} / \mathrm{Hz}\right)
$$

and that results in a power spectral density of the mass acceleration of

$$
\Phi_{B}(f)=\frac{f^{4}}{\left(f_{0}^{2}-f^{2}\right)^{2}+\left(2 \xi f_{0} f\right)^{2}} \frac{16 \pi k_{B} T \xi f_{0}}{m}\left(\mathrm{~m} / \mathrm{s}^{2}\right)^{2} / \mathrm{Hz}
$$

where $k_{B}=1.38310223 \times 10^{-23} \mathrm{~J} / \mathrm{K}$ is the Boltzmann's constant, $T$ is the temperature, $f_{0}$ is the resonance frequency of the oscillator, and $\xi$ is the percentage of critical damping. For example, taking $\xi=0.7, T=300 \mathrm{~K}, m=10 \mathrm{gr}, f_{0}=$ $1 \mathrm{~Hz}$ gives $\Phi_{B}(f)=7.3 \times 10^{-17}\left(\mathrm{~m} / \mathrm{s}^{2}\right)^{2} / \mathrm{Hz}$, which corresponds to an rms displacement of $0.02 \mathrm{~nm}$. This contribution becomes larger with a decreasing size of the proof mass.

Thermoelectrical noise, or Johnson noise, is the electrical equivalent of the thermomechanical noise. It is generated in the resistive components of the sensor circuit due to random thermal excitation of the charge carriers, generating a white noise voltage of

$$
\Phi_{J}(f)=4 k_{B} T R\left(V^{2} / \mathrm{Hz}\right),
$$

where $R$ is the resistance. Typically, it corresponds to an rms value of some picometers above one hertz. For a capacitor $C$, the dielectric loss can be modeled by placing a resistance $R_{C}$ in parallel to the capacitor (van der Poel, 2010). The equivalent resistor is

$$
R_{C}=\frac{1}{\eta \omega C},
$$

where $\eta$ is the loss factor of the capacitor.

Shot noise, or Schottky noise, is caused by random movement of electric charges across potential barriers, such as $\mathrm{p}-\mathrm{n}$ junctions (Pallas-Areny and Webster, 2001). Its power spectral density $\Phi_{\mathrm{sh}}(f)$ can be modeled by

$$
\Phi_{\mathrm{sh}}(f)=2 q I_{\mathrm{dc}}\left(A^{2} / \mathrm{Hz}\right),
$$

where $I_{\mathrm{dc}}$ is the average current that crosses the barrier and $q$ is the electric charge. It has the shape of a white-noise current.

Flicker noise, or $1 / f$ noise, is mainly due to impurities in the production of semiconductor devices. Its main characteristic is that its power spectral density is inversely proportional to the frequency and can be modeled by

$$
\Phi_{V}(f) \mid=K / f^{\alpha}\left(V^{2} / \mathrm{Hz}\right)
$$

where the exponent is usually $\alpha=1$. Typical values for sensor amplifier's noise constants can be found in van der Poel (2010).

As these sources of noise are uncorrelated, the overall sensor noise floor $\Phi_{n n}(f)$ can be directly estimated by summing all of the contributions using consistent units:

$$
\Phi_{n n}(f)=\Phi_{B}(f)+G^{2}\left\{\Phi_{J}(f)+Z^{2}(f) \Phi_{\mathrm{sh}}(f)+\Phi_{V}(f)\right\},
$$

where $Z(f)$ is the load at which the current noise is developed and $G$ is the sensitivity, converting the voltage $(V)$ into acceleration $\left(\mathrm{m} / \mathrm{s}^{2}\right)$. The next section provides a tool to evaluate the magnitude of the overall noise arising from a set of sensors and amplifiers.

\section{Detection}

Let us call $U$ the quantity to measure (see Fig. 14). Place two sensors of the same type side by side, with $S_{1}$ and $S_{2}$ as their respective sensitivities.

Assuming that $N_{1}$ and $N_{2}$ are the noise of the two sensors, the outputs in the frequency domain are given by

$$
X(\omega)=S_{1}(\omega) U(\omega)+N_{1}(\omega)
$$

and

$$
Y(\omega)=S_{2}(\omega) U(\omega)+N_{2}(\omega) .
$$

Resolving equation (52) for $U(\omega)$ and substituting the solution into equation (53) gives

$$
Y(\omega)=H(\omega) X(\omega)+N(\omega),
$$

where $\quad H(\omega)=S_{2}(\omega) / S_{1}(\omega) \quad$ and $\quad N(\omega)=N_{2}(\omega)-$ $H(\omega) N_{1}(\omega)$. If $N_{1}$ and $N_{2}$ are statistically independent of each other and of the quantity $U$, it follows that

$$
\Phi_{y y}=H^{2} \Phi_{x x}+\Phi_{n n}=\Phi_{s s}+\Phi_{n n},
$$

which expresses that the total signal power spectral density is the sum of a coherent useful signal $\Phi_{s s}$ and noise $\Phi_{n n}$.

In practice, from the two time histories $x(t)$ and $y(t)$, we first calculate the power spectral densities of each signal and the transfer function $H(\omega)$ between them:

$$
H(\omega)=\frac{\Phi_{x y}}{\Phi_{x x}} .
$$

Then, $\Phi_{s s}$ is calculated by

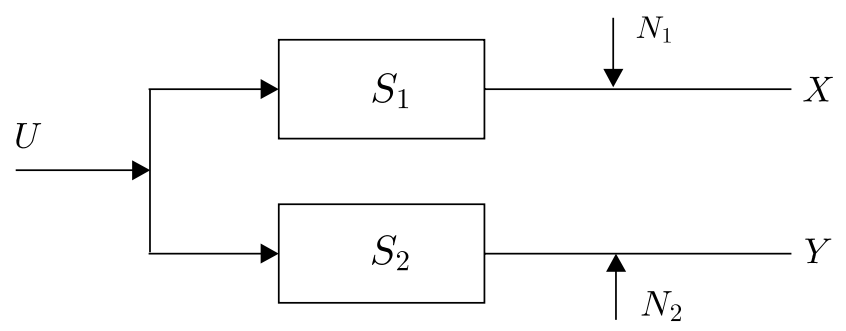

Figure 14. Two sensors measuring the same input vibration (Holcomb, 1989; Montag, 1996a,b). 


$$
\Phi_{s s}=H^{2} \Phi_{x x},
$$

and $\Phi_{n n}$ is calculated by equation (55). The coherence $\gamma_{x y}^{2}$ between $x(t)$ and $y(t)$ is defined as the ratio between $\Phi_{s s}$ and $\Phi_{y y}$,

$$
\gamma_{x y}^{2}(\omega)=\frac{\Phi_{x y}^{2}(\omega)}{\Phi_{x x}(\omega) \Phi_{y y}(\omega)}
$$

the signal-to-noise ratio $(\beta)$ is the ratio between $\Phi_{s s}$ and $\Phi_{n n}$,

$$
\beta(\omega)=\frac{\Phi_{s s}}{\Phi_{n n}}=\frac{\Phi_{x x} H^{2}}{\Phi_{n n}}=\frac{\gamma_{x y}^{2}}{1-\gamma_{x y}^{2}},
$$

and the ratio between the amplitudes of signal and noise is $\sqrt{\beta}$. The amplitude spectrum of the noise is

$$
N(\omega)=\Phi_{n n}^{1 / 2}(\omega)
$$

and its rms value is

$$
N_{\mathrm{rms}}=\left[\int_{0}^{\infty} \Phi_{n n}(\nu) d \nu\right]^{1 / 2}
$$

Finally, using equations (60) and (2), we can readily evaluate the resolution $R$ of the sensor. Figure 15 compares the $R$ of a few different sensors: geophone (Mark L4C), seismometer (Güralp CMG-6T), capacitive (PI PISECA 510), and interferometric (Acernese et al., 2010; Braun and Karrai, 2011). Except for the curve corresponding to the interferometric sensor (which has been fitted from data taken in the corresponding references), the curves have been fitted from experimental data using the method described previously in section Sources of Internal Noise. At low frequency, the $1 / f$ noise is inherent to the electronic circuitry. At higher frequency, capacitive sensors have a resolution about 10 times better than magnetic sensors, and interferometric sensors further improve the resolution by another factor of 10 . Of course, the exact location of the curves depends on the sensor used, but the tendencies should be conserved.

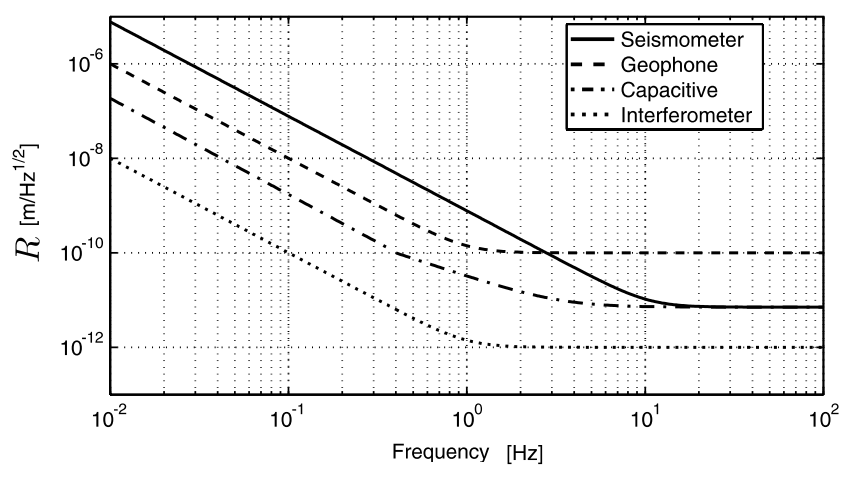

Figure 15. Comparison of the sensor noise floors.

\section{Conclusions}

In this paper, the main types of inertial sensors used to measure low-frequency linear vibrations have been reviewed: displacement sensors, geophones, accelerometers, and seismometers. The working principle of each has been explained, along with their main characteristics. In summary, all of them use a single-degree-of-freedom oscillator as absolute reference. Typically, the oscillator has a resonance frequency of a few hertz. However, geophones use a coil as the sensitive element, while displacement sensors and seismic accelerometers use a relative displacement sensor. For both accelerometers and geophones, the dynamic range can be extended using the force-balance principle. Usually, accelerometers are more suitable to measure low-frequency signals because their bandwidth corresponds to frequencies lower than the resonance of the inertial mass. Geophones and displacement sensors are more suitable to measure highfrequency signals because their bandwidth corresponds to frequencies higher than the resonance of the inertial mass. For each sensor type, the procedure to extend the bandwidth by moving the corner frequency has also been presented. Then, the primary fundamental limitations of inertial sensors have been reviewed: tilt-to-horizontal coupling, zero-length springs, and sources of noise. Finally, the procedure to evaluate the overall sensor noise has been presented, along with numerical examples, showing that optical sensors show currently the best noise figure, and a high potential for the development of future inertial sensors (Fig. 15).

\section{Data and Resources}

A comparison of commercial accelerometers and seismometers can be found in http://www.passcal.nmt.edu/content /instrumentation/sensors/sensor-comparison-chart (last accessed November 2011). All data used in this paper came from the published sources listed in the references.

\section{Acknowledgments}

The research leading to these results has been jointly funded by the European Commission under the FP7 Research Infrastructures project EuCARD, Grant Agreement No. 227579 and by the Brussels Capital Region under the program Brain Back to Brussels for the first author. The authors gratefully acknowledge the reviewers for their useful comments to improve the quality of this manuscript.

\section{References}

Acernese, F., G. Giordano, R. Romano, R. De Rosa, and F. Barone (2008). Mechanical monolithic horizontal sensor for low frequency seismic noise measurement, Rev. Sci. Instrum. 79, 074501-1-074501-8.

Acernese, F., G. Giordano, R. Romano, R. De Rosa, and F. Barone (2010). Tunable mechanical monolithic sensor with interferometric readout for low frequency seismic noise measurement, Nucl. Instrum. Meth. Phys. Res. A 617, 457-458.

Barzilai, A., T. VanZandt, and T. Kenny (1998). Technique for measurement of the noise of a sensor in the presence of large background signals, Rev. Sci. Instrum. 69, 2767-2772. 
Barzilai, A., T. VanZandt, T. Pike, S. Manionand, and T. Kenny (1998). Improving the performances of a geophone through capacitive position sensing and feedback, in American Society of Mechanical Engineers International Congress, International ASME Congress on Dynamic systems and control, Anaheim, California, USA, 15-20 November 1998.

Bertolini, A., N. Beverini, G. Cella, R. DeSalvo, F. Fidecaro, M. Francesconi, and D. Somonetti (2004). Geometric anti-spring vertical accelerometers for seismic monitoring, Nucl. Instrum. Meth. Phys. Res. A 518, 233-235.

Bertolini, A., G. Cella, R. DeSalvo, and V. Sannibale (1999). Seismic noise filters, vertical resonance frequency reduction with geometric antisprings: A feasibility study, Nucl. Instrum. Meth. Phys. Res. A 435, 475-483.

Bertolini, A., R. DeSalvo, F. Fidecaro, M. Francesconi, S. Marka, V. Sannibale, D. Simonetti, A. Takamori, and H. Tariq (2006a). Mechanical design of a single-axis monolithic accelerometer for advanced seismic attenuation systems, Nucl. Instr. Meth. Phys. Res. A 556, 616-623.

Bertolini, A., R. DeSalvo, F. Fidecaro, M. Francesconi, S. Marka, V. Sannibale, D. Simonetti, A. Takamori, and H. Tariq (2006b). Readout system and predicted performance of a low-noise low-frequency horizontal accelerometer, Nucl. Instrum. Meth. Phys. Res. A 564, 579-586.

Braun, P.-F., and K. Karrai (2011). Multi-channel optical fiber based displacement metrology, in Proc. of the 11th Euspen International Conference, Como, Italy, 23-27 May 2011, 54-57.

Barzilai, A. (2000). Improving a geophone to produce an affordable, broadband seismometer, Ph.D Thesis, Stanford University.

Cacho, S., P. Lognonne, J. F. Karczewski, G. Pont, and G. Coste (1999). A very broad band 3 axis seismometer to study internal structure of Mars, in Space Mechanisms and Tribology, Proc. of the 8th European Symposium Space Mechanisms and Tribology, Toulouse, France, 29 September-1 October 1999.

Collette, C., K. Artoos, A. Kuzmin, S. Janssens, M. Sylte, M. Guinchard, and C. Hauviller (2010). Active quadrupole stabilization for future linear particle colliders, Nucl. Instrum. Meth. Phys. Res. A 621, 71-78.

Daku, B. L. F., E. M. A. Mohamed, and A. F. Prugger (2004). A PVDF transducer for low-frequency acceleration measurements, ISA Trans. 43, 319-328.

Faber, K., and P. W. Maxwell (1997). Geophone spurious frequency: What is it and how does it affect seismic data quality? Can. J. Explor. Geophys. 33, 46-54.

Fraanje, P. R., N. Rijnveld, and T. C. Van den Dool (2009). A vibration sensor and a system to isolate vibrations, WO Patent No. 2009/139628 A1, http://patentscope.wipo.int/search/en/WO2009139628 (last accessed 26 May 2012).

Frisch, J., V. Decker, E. Doyle, L. Hendrickson, T. Himel, T. Markiewicz, and A. Seryi (2004). Development of a non-magnetic inertial sensor for vibration stabilization in a linear collider, in 22nd International Linear Collider Conference, Lubeck, Germany, 16-20 August 2004.

Frisch, J., E. Doyle, L. Eriksson, L. Hendrickson, T. Himel, T. Markiewicz, and R. Partridge (2003). Inertial sensor development for active vibration stabilization, in IEEE Particle Accelerator Conference, Portland, Oregon, U.S.A., 12-16 May 2003.

Gardner, D., T. Hofler, S. Baker, R. Yarber, and S. Garrett (1987). A fiber-optic interferometric seismometer, J. Lightwave Tech. 5, 953-960.

Holcomb, L. G. (1989). A direct method for calculating instrument noise levels in side-by-side seismometer evaluations, U.S. Dept. Int. Geol. Surv. Tech. Rept. 89-214, 35 pp.

Kittel, C. (1958). Elementary Statistical Physics, John Wiley, New York, USA.

Kollias, A. T., and J. N. Avaritsiotis (2005). A study of the performance of bending mode piezoelectric accelerometers, Sensor Actuator Phys. 121, 434-442.

LaCoste, L. J. B. (1934). A new type of long period seismograph, Physics $\mathbf{5}$, no $7,178-180$.
Laro, D., S. van den Berg, J. Eisinger, and J. van Eijk (2011). 6-dof active vibration isolation without tilt-horizontal coupling, in Proc. of the 11th Euspen International Conference, Como, Italy, 23-27 May $2011,15-18$

Lee, W. H. K., M. Celebi, M. I. Todorovska, and H. Igel (Guest Editors) (2009). Rotational Seismology and Engineering Applications, special issue, Bull. Seismol. Soc. Am. 99, no. 2B.

Ling, F. H., W. M. Wang, and R. J. Mao (1990). Dynamic stability analysis of the geophone spurious frequency phenomenon, J. Sound Vib. 139, $21-30$.

Liu, C.-H., and T. W. Kenny (2001). A high precision, wide-bandwidth micromachined tunneling accelerometer, in IEEE ASME J. Microelectromech. Syst. 10, 425-433.

Melton, B. S., and D. P. Johnson (1962). Inertial seismograph designLimitations in principle and practice (or how not to build a sensitive seismograph), in Proceedings of the Institute of Radio Engineers, 2328-2339.

Montag, C. (1996a). Active stabilization of mechanical quadrupole vibrations for linear collider, Nucl. Instrum. Meth. Phys. Res. A 378, 396-375.

Montag, C. (1996b). Active stabilization of mechanical quadrupole vibrations in a linear collider test facility, Ph.D Thesis, Hamburg University.

Oome, A. J. J. A., J. L. G. Janssen, L. Encica, E. Lomonova, and J. A. A. T. Dams (2009). Modeling of an electromagnetic geophone with passive magnetic spring, Sensor Actuator Phys. 153, 142-154.

Pallas-Areny, R., and J. G. Webster (2001). Sensors and Signal Conditioning, John Wiley, New York, USA.

Pazos, A., G. Alguacil, and J. Martin Davila (2005). A simple technique to extend the bandwidth of electromagnetic sensors, Bull. Seismol. Soc. Am. 95, 1940-1946.

Plesinger, A. (1984). Analysis and optimization of wide-band force-balance seismometer responses, Studia Geophysica et Geodaetica 28, 67-81.

Preumont, A. (2006). Mechatronics, Dynamics of Electromechanical and Piezoelectric Systems, Springer, Dordrecht, The Netherlands.

Rijnveld, N., and T. C. Van Den Dool (2010). An active vibration isolation and damping system, WO Patent No. 2010/143959, http://patentscope .wipo.int/search/en/WO2010143959 (last accessed 26 May 2012).

Rodgers, P. W. (1968). The response of the horizontal pendulum seismometer to Rayleigh and Love waves, tilt, and free oscillations of the Earth, Bull. Seismol. Soc. Am. 58, 1385-1406.

Rodgers, P. W. (1969). A note on the response of the pendulum seismometer to plane wave rotation, Bull. Seismol. Soc. Am. 59, 2101-2102.

Rodgers, P. W. (1992). Frequency limits for seismometers as determined from signal-to-noise ratios. Part 1: The electromagnetic seismometer, Bull. Seismol. Soc. Am. 82, 1071-1098.

Rodgers, P. W. (1993). Maximizing the signal-to-noise ratio of the electromagnetic seismometer: The optimum coil resistance, amplifier characteristics, and circuit, Bull. Seismol. Soc. Am. 83, 561-582.

Rodgers, P. W., A. M. Martin, M. C. Robertson, M. M. Hsu, and D. B. Harris (1995). Signal-coil calibration of electromagnetic seismometers, Bull. Seismol. Soc. Am. 85, 845-850.

Roset, X., J. del Rio, A. Manuel, and R. Palomera-Garcia (2004). Contributions to model characterization of geophone sensor, in IEEE Instrumentation and Measurement Technology Conf., Como, Italy, 18-20 May 2004, 1896-1899.

Rowan, S., J. Hough, and D. R. M. Crooks (2005). Thermal noise and material issues for gravitational wave detectors, Phys. Lett. 347, 25-32.

Saulson, P. R. (1990). Thermal noise in mechanical experiments, Phys. Rev. 42, 2437-2445.

Sheffield, H. E (1964). An electronic vertical long-period seismometer, IEEE Trans. Inst. Meas. 13, 2-7.

Teupser, C., and A. Plesinger (1979). Design of feedback-controlled wideband seismographs with respect to undesired side-effects, Phys. Earth Planet In. 18, 58-63.

Usher, M. J., R. F. Burch, and C. Guralp (1979). Wide-band feedback seismometers, Phys. Earth Planet In. 18, 38-50.

van der Poel, G. W. (2010). An exploration of active hard mount vibration isolation for precision equipment, Ph.D Thesis, University of Twente. 
van der Poel, T., J. van Dijk, B. Jonker, and H. Soemers (2007). Improving the vibration isolation performance of hard mounts for precision equipment, in IEEE ASME International Conference on Advanced Intelligent Mechatronics, Zurich, Switzerland, 4-7 September 2007.

Wielandt, E., and G. Streckeisen (1982). The leaf spring seismometer: Design and performance, Bull. Seismol. Soc. Am. 72, 2349-2367.

Wielandt, E. (2002). Seismometry, in International Handbook of Earthquake and Engineering Seismology, Part A, W. H. K. Lee, H. Kanamori, P. C. Jennings, and C. Kisslinger (Editors), Academic Press, Amsterdam, The Netherlands, 283-304.

Zumberge, M., J. Berger, J. Otero, and E. Wielandt (2010). An optical seismometer without force feedback, Bull. Seismol. Soc. Am. 100, 598-605.

Zuo, L. (2004). Element and system design for active and passive vibration isolation, Ph.D Thesis, Massachusetts Institute of Technology.

Zuo, L., and S. Nayfeh (2004). An integral sliding control for robust vibration isolation and its implementation, in Smart Structures and Materials 2004: Damping and Isolation, K. -W. Wang (Editor), Vol. 5386, Proceedings of the SPIE, 1-10, doi 10.1117/12.539616.
European Organization for Nuclear Research, CERN

Engineering Department

Geneva 23

1211 Geneva

Switzerland

(C.C., S.J., P.F.-C., K.A., M.G., C.H.)

University of Brussels

Active Structures Laboratory

50 av. Roosevelt

1050 Brussels

Belgium

(A.P.) 